\title{
Target Genes of Circulating miR-34c as Plasma Protein Biomarkers of Alzheimer's Disease and Mild Cognitive Impairment
}

\author{
Arin L Zirnheld ${ }^{1}$, Erik L Regalado ${ }^{1}$, Vikranth Shetty ${ }^{1}$, Howard Chertkow ${ }^{2,3,4,5}$, Hyman M Schipper $2,3,4,5$ and Eugenia Wang ${ }^{1 *}$ \\ ${ }^{1}$ Advanced Genomic Technology, LLC, 201 E. Jefferson St., Louisville, Kentucky, USA \\ ${ }^{2}$ Department of Neurology \& Neurosurgery, McGill University, Montréal, Québec, Canada \\ ${ }^{3}$ Memory Clinic, Jewish General Hospital, Montréal, Canada \\ ${ }^{4}$ Bloomfield Centre for Research in Aging, Lady Davis Institute, Montréal, Canada
}

${ }^{5}$ Department of Clinical Neurosciences and Division of Geriatric Medicine of the Jewish General Hospital, Montréal, Québec, Canada

${ }^{*}$ Corresponding author: Wang E, Advanced Genomic Technology, LLC, 201 E. Jefferson St., Louisville, Kentucky, USA 40202US Highway 42, Unit 433, Louisville, Kentucky, 40241, USA, Tel: (502) 228-5438; E-mail: EWangAGT@gmail.com

Received date: July 07, 2015; Accepted date: August 18, 2015; Published date: August 25, 2015

Copyright: ( 2015 Zirnheld AL, et al. This is an open-access article distributed under the terms of the Creative Commons Attribution License, which permits unrestricted use, distribution, and reproduction in any medium, provided the original author and source are credited.

\begin{abstract}
MicroRNAs repress post-transcriptional expression of a variety of genes, several of which are involved in neurological development and Alzheimer's disease (AD) pathology. In previous studies, we have shown that plasma miR-34c and miR-34a levels are increased in probable AD patients. In this study, we show that four key gene products silenced by these miRNAs, Onecut homoebox 2 (ONECUT2), B-cell lymphoma (BCL2), sirtuin 1 (SIRT1), and presenilin 1 (PSEN1), are all decreased in abundance in plasma specimens from mild cognitive impairment $(\mathrm{MCl})$ and probable $A D$ individuals. Additionally, there is a statistically significant inverse correlation between levels of miR-34c and expression of all four proteins, and between miR-34a and ONECUT2 expression. In particular, our results suggest that ONECUT2 plasma levels may act as a novel biomarker for early stages of cognitive decline. Additionally, we find statistically significant differences in expression levels of the other three targets, BCL2, SIRT1, and PSEN1, in circulating plasma, and levels of these proteins effectively distinguish among all three groups: normal elderly controls (NEC), $\mathrm{MCl}$, and probable AD. Therefore, the inverse relationship between miR-34c and miR-34a and their targets may initially be detected by early decline in ONECUT2 expression, upon entry to the MCI stage, followed by decreased BCL2, SIRT1 and PSEN1 expression as patient's transition to full-blown AD dementia.
\end{abstract}

Keywords: Alzheimer's disease; BCL2; SIRT1; Presenilin-1; ONECUT2; Mild cognitive impairment

\begin{abstract}
Abbreviations:
AD: Alzheimer's Disease; MCI: Mild Cognitive Impairment; NEC: Normal Elderly Controls; A $\beta 42$ : Amyloid Beta 42; PSEN1: Presenilin-1; BCL2: B-cell Lymphoma 2; SIRT1: Sirtuin 1; ONECUT2: Onecut Homeobox 2; MMSE: Mini-Mental State Exam; LSD: Fisher's Least Significant Difference Statistic; ROC: Receiver Operating Characteristic; AUC: Area Under the Curve

\section{Introduction}

An estimated 24.3 million people worldwide have $\mathrm{AD}$, and it is projected that the number of $\mathrm{AD}$ sufferers will double every 20 years until 2040 [1]. AD pathology is characterized by the presence of extracellular plaques composed of amyloid beta fragments and intracellular neurofibrillary tangles composed of hyper phosphorylated tau [2]. Furthermore, mutations in amyloid precursor protein (APP) and its cleavage products, amyloid beta $40\left(\mathrm{~A} \beta_{40}\right)$ and amyloid beta $42\left(\mathrm{~A} \beta_{42}\right)$, and mutant PSEN1 and PSEN2, both involved in aberrant APP cleavage, are associated with development of familial $\mathrm{AD}$ [3]. Although the presence of plaques and tangles are indicative of $\mathrm{AD}$, a variety of other mechanisms participate in disease pathogenesis, including inflammation, excitotoxicity, oxidative stress, synaptic dysfunction, and apoptosis $[2,4]$.
\end{abstract}

The post-transcriptional repression by a particular microRNA of its target gene's expression usually results in the decreased expression of the protein, i.e. increased abundance of a miRNA corresponds to decreased presence of its target protein(s). This effect is observed not only in in vitro functional assays in cultured cell systems, but also at the physiological level in numerous tissue and disease states. We have demonstrated such reciprocal expression with increased miR-34a expression and decreased abundance of its target genes in circulating mouse plasma during aging [5]. Recently, we demonstrated that miR-34a and its sister, miR-34c, are increased in abundance in plasma from patients with probable $\mathrm{AD}$ [6]. In particular, we showed that miR-34c is capable of repressing the expression of key target genes including ONECUT2, SIRT1, BCL2, and PSEN1, all of which are known to be involved in $\mathrm{AD}$-related pathogenesis [6].

Target genes of miR-34c examined in our study are involved in the upstream regulation of neuronal development, apoptosis, and protection from oxidative stress, neuronal survival, and $A \beta 42$ production. For example, ONECUT2, expressed in several cell types including neurons, is involved in development and differentiation of motor and sensory neurons, as well as the development of several brain regions [7-11]. SIRT1 has a protective, anti-apoptotic effect in cells, perhaps through protection from oxidative stress, and also provides protection from tau hyper phosphorylation [12-20]. BCL2, an anti-apoptotic, pro-survival cellular protein associated with tumor genesis [21], is elevated during brain development [22], remains relatively stable in certain areas of the brain during adulthood, and then declines during aging, leading to decreased neuronal survival 
[23]. Additionally, $A \beta_{42}$ can suppress BCL2 activity, resulting in increased apoptosis [24,25], and overexpression of BCL2 results in suppression of $\mathrm{A} \beta_{42}$-induced apoptosis [26]. Finally, mutations in the protein PSEN1, a component of the machinery involved in APP cleavage [27], are associated with the development of early-onset familial $\mathrm{AD}[28,29]$ as well as late-onset sporadic $\mathrm{AD}[30]$.

Therefore, the paradigm for our study is to determine whether the observed increase of miR-34c in circulating plasma of AD patients is accompanied by the reduced presence of key target genes ONECUT2, SIRT1, BCL2, and PSEN1, all of which are functionally implicated in the cognitive decline associated with Alzheimer's disease. Interestingly, we find significantly decreased levels of ONECUT2, SIRT1, BCL2, and PSEN1 in AD and MCI plasma, implicating these proteins as potential biomarkers for neurodegeneration. Additionally, a statistically significant inverse correlation exists between miR-34c plasma levels and levels of ONECUT2, SIRT1, BCL2, and PSEN1, and between miR-34a plasma levels and ONECUT2 plasma abundance over the course of disease progression. Statistical analysis indicates that ONECUT2 levels may be used to differentiate between NEC and MCI, and between NEC and probable AD, while SIRT1, BCL2, and PSEN1 levels effectively distinguish all three states: NEC, MCI, and AD. Overall, plasma levels of these four proteins may be excellent biomarkers for the transition from normalcy to mild cognitive impairment and finally progression to $\mathrm{AD}$-associated dementia.

\section{Materials and Methods}

\section{Subject identification and enrollment}

Subjects recruited for this study consisted of individuals aged 60 to 85 , with 10 or more years of education. Those diagnosed with $\mathrm{AD}$ or MCI were recruited to the study at the Memory Clinic of the Jewish General Hospital (JGH) in Montréal, and normal community elderly volunteers were recruited through advertising. Subjects received standardized clinical, mental status, neuropsychological, and cognitive evaluation. Depression was excluded via the Yesavage Geriatric Depression Scale (Geriatric Depression scale>15) [31]. None had evidence of cerebral infarct on CT, and all subjects scored less than 4 on the Hachinski ischemic scale [32]. No individuals accepted for the study had evidence of significant heart disease, alcoholism, or drug use, and all showed normal routine blood work.

Subjects were classified as normal if they lacked subjective memory complaints and scored at or above 26 on the Montreal Cognitive Assessment (MoCA) test [33] and above 25 on the Folstein MiniMental State Exam (MMSE) [34].

The AD patients received diagnoses of mild, moderate, or severe probable AD according to standard clinical criteria [35,36]. They scored below 26 on the MoCA [33], between 4 and 25 on the MMSE [34], and showed significant functional impairment on the LawtonBrody functional scale [37].

Subjects with MCI met the standard criteria for MCI defined by the working group of Winblad et al. [38], as well as by Petersen in his original operational definition of MCI $[39,40]$. All had a history of memory decline in the last 1-4 years, reported by the patient, caregiver (usually the spouse), or both, of a sufficient degree to bring them to medical attention. All subjects scored below 26 on the MoCA [33]. They were also documented to have objective memory impairment on a standardized mental status exam, the JGH Memory Clinic Assessment (unpublished), which contains elements of the CERAD,
CDR, and Toronto Behavioural Neurology Assessment batteries appropriate for mild dementia subjects [41]. In the clinical judgement of the specialists assessing these individuals, they exhibited "memory impairment beyond what was felt to be normal for age, but were relatively intact in other cognitive domains" [42]. Thus, the diagnosis of MCI, and indeed amnestic MCI, was primarily a clinical one. The subjects did not meet the NINCDS-ADRDA criteria for the diagnosis of probable AD or the DSM-3 criteria for dementia, due to the lack of other significant associated cognitive deficits or to the lack of impairment in daily functioning [35,43]. None showed significant functional impairment on the Lawton-Brody functional scale [37].

The diagnoses of AD or MCI were supported by detailed neuropsychological testing containing the following elements: Montréal Cognitive Assessment test [33], Modified WAIS subtests (digit symbol) [44], Wechsler Memory Scale-R subtests (Logical Memory, Digit Span) [45], Ravens Progressive Matrices $[46,47]$ Visual perceptual tests, RAVLT $[48,49]$, as well as Verbal Fluency.

\section{Sample collection, storage, and shipment}

A thirty milliliter blood sample was collected from each participant in an EDTA Vacutainer( $)$ at the JGH. The samples were drawn from the antecubital vein with an 18-24 gauge butterfly or 22 gauge needles. Within three hours, plasma was isolated using Ficoll-Paque Plus (GE Healthcare, Piscataway, NJ), and stored in RNAlater buffer solution (Ambion, Austin, TX). Samples were shipped to the Advanced Genomic Technology laboratory in Louisville, Kentucky, where they were stored at $-80^{\circ} \mathrm{C}$ in $200 \mathrm{ul}$ aliquots. Sample collection and recruitment of individuals participating in this study were approved by IRB protocol \#09-131 of the Research Ethics Committee of the Jewish General Hospital in Montréal, Québec, Canada.

\section{RNA isolation, cDNA synthesis, and quantitative PCR (qPCR)}

RNA was extracted using Qiagen's miRNeasy serum/plasma kit (Qiagen, Venlo, Limberg, Netherlands). Aliquots of each sample were thawed on ice and spun in a microcentrifuge for 3 minutes at $3000 \mathrm{x}$ g. The protocol for RNA extraction followed the vendor's product handbook. The organic phase was saved for further protein extraction; the concentration of the resultant RNA was measured with a NanoVue (GE Healthcare Life Sciences, Pittsburgh, PA).

Complementary DNA specific to each target miRNA or endogenous control miRNA was synthesized by adding 100-200 ng of RNA to a cDNA reaction using the Taqman microRNA Reverse Transcription kit (Life Technologies, Carlsbad, CA) with the 5X primers provided in the Taqman small RNA assays (Life Technologies) for each miRNA. Reactions were placed in a Veriti 96 well Thermalcycler (Life Technologies), according to the protocol provided in the Taqman small RNA assay protocol handbook.

Complementary DNA template $(1.33 \mathrm{ul})$ was added to each qPCR reaction well with $20 \mathrm{X}$ probes provided in the Taqman small RNA assays (Life Technologies) and real-time qPCR EvaGreen Master Mix (MidSci, St. Louis, MO) in triplicate. The plates were loaded into either an ABI7500 or ABI7500 Fast Real-time PCR system (Life Technologies). 


\section{Protein isolation, electrophoresis, and Western blotting}

Using $100 \%$ isopropanol, proteins were precipitated from the organic phase left behind after removal of the aqueous phase during RNA isolation. The samples were spun and the pellet washed with 0.3 M guanidine hydrochloride in $95 \%$ ethanol three times. The pellets were then incubated for 20 minutes in $100 \%$ ethanol, and transferred to a new tube. Pellets were dried for 15 minutes, dissolved in $1 \%$ SDS, and incubated at $55^{\circ} \mathrm{C}$ for 2-3 hours [50].

Protein concentrations were determined by the Bradford method. Albumin and gamma-globulin proteins were then removed from $500-600 \mu \mathrm{g}$ of protein using an albumin and IgG removal kit (Pierce, Rockford, IL). Protein concentrations were again determined by the Bradford method, and samples were stored at $-80^{\circ} \mathrm{C}$.

Following isolation, protein extracts were run on $10.5 \%$ polyacrylamide SDS-PAGE gels, and transferred to nitrocellulose membranes for $2-3$ hours in $20 \%$ methanol transfer buffer. Membranes were stained with Ponceau Red to visualize total protein transfer and for normalization of the loaded protein samples. Membranes were then blocked in 5\% milk or bovine serum albumin for 1 hour, incubated in the appropriate primary antibody overnight at $4^{\circ} \mathrm{C}$, washed in Tris-Buffered Saline-Tween (Sigma-Aldrich, St. Louis, $\mathrm{MO}$ ) and incubated in the secondary antibody for 1-2 hours at room temperature. Membranes were again washed and incubated in ECL solution (Pierce) for 2 minutes, and then imaged using autoradiography.

\section{Data analysis}

Relative expression values for qPCR data were calculated using the $1 / \Delta \mathrm{Ct}$ method. Mean intensity values for Western blot data were calculated using ImageJ software (Public Domain, NIMH, NIH, Bethesda, MD) to find the intensity of each target protein band, which was then normalized by dividing this value by the intensity of the corresponding Ponceau-stained band. Although a housekeeping gene, such as $\beta$-actin or tubulin, is often used to normalize Western blot data from tissue or cultured cells, the abundance of these proteins is not well-characterized in cell-free plasma. Additionally, these housekeeping proteins may not be suitable for all systems or conditions, and may not always accurately reflect protein concentration [51-53]. Furthermore, it is unknown whether various disease states may induce changes in expression of these genes. A band recognized by Ponceau staining with consistent intensity across all lanes has been reported as a suitable alternative to the use of housekeeping genes for Western blot normalization [54]. All normalized values were then averaged, and the mean intensity was reported. Intensities were compared among the NEC, MCI, and AD cohorts using a Fisher's Least Significant Difference (LSD) test [55]. Correlation analysis was performed using SPSS statistical software. Pearson correlation values ( $\mathrm{R}$ ) and $\mathrm{p}$ values were calculated. A p-value of less than 0.05 indicates a significant relationship.

Data were further subjected to Receiver Operating Characteristic (ROC) analysis to determine the sensitivity, specificity and accuracy of the biomarkers [56]. ROC curve analysis is used to compare results of a diagnostic laboratory test for two or more groups. The ROC curve is plotted using sensitivity (which represents true positive values) on the $y$-axis against 1-Specificity (which represents false positive values) on the $\mathrm{x}$-axis. The resulting area under the curve (AUC) demonstrates the overall ability of the test to discriminate between the two groups compared. AUC values range from 0.5 to 1 . A bad test has an area of
0.5 , which is close to the diagonal line, and an area of 1.00 indicates perfect accuracy [56-58]. Calculation of all parameters is done using formulas described in our previous paper [6].

\section{Results}

\section{Patient demographics and genetics}

Ten samples were selected from each cohort (AD, MCI and NEC) to match age and years of education among the three groups. The mean age was 71.7 for the NEC samples, 74.3 for the MCI group, and 82.0 for the AD group (Table 1). Mean years of education were similar among the three groups, with an average of 14.3 years in the NEC group, 13.3 in the $\mathrm{MCI}$ group, and 13.0 in the AD subjects. There were differences in the percentage of participants possessing at least one ApoE4 allele: $36.4 \%$ of AD samples had either a $3 / 4$ or $4 / 4$ genotype; $8.3 \%$ of the MCI group had either a $3 / 4$ or $4 / 4$ genotype; and the NEC group contained no subjects with either genotype. This statistic reflects the composition of our bio bank: more $\mathrm{AD}$ and $\mathrm{MCI}$ patients possess an ApoE4 allele than participants from the NEC group, reflecting the important role of the Apoe4 allele as an AD risk factor [59].

\begin{tabular}{|c|c|c|c|}
\hline & NEC & MCI & AD \\
\hline N & 10 & 10 & 10 \\
\hline Mean Age (S.D) & $71.7(3.1)$ & $74.3(9.0)$ & $82.0(6.9)$ \\
\hline Mean Education (S.D) & $14.3(3.8)$ & $13.3(3.8)$ & $13(4.7)$ \\
\hline \% ApoE 4/4 or 3/4 positive & 0 & 8.3 & 36.4 \\
\hline
\end{tabular}

Table 1: Characteristics of NEC, MCI, and AD subjects. Demographic parameters, including age, education, and ApoE allele status are presented for each cohort examined in the study.

\section{Expression of ONECUT2, SIRT1, BCL2, and PSEN1 in AD and NEC plasma}

Our previous data indicate that miR-34c constructs repress expression of ONECUT2, SIRT1, BCL2, and PSEN1, when transfected into HEK293 cells [6]. In the current study, we first measured levels of these four proteins in plasma from both AD and NEC participants, by Western blot (Figure 1A). We found the average expression of each protein to be significantly reduced in the $\mathrm{AD}$ cohort compared to the NEC subjects (Figure 1B and Table 2).

\begin{tabular}{|c|c|c|}
\hline & AD & NEC \\
\hline Protein & Avg (S.D) & Avg (S.D) \\
\hline BCL2 & $1.259(0.08)$ & $1.522(0.08)$ \\
\hline PSEN1 & $0.767(0.09)$ & $0.955(0.10)$ \\
\hline ONECUT2 & $0.626(0.02)$ & $0.763(0.02)$ \\
\hline SIRT1 & $0.816(0.01)$ & $0.842(0.02)$ \\
\hline
\end{tabular}

Table 2: Average relative intensities of protein expression in AD and NEC subjects. Relative intensities for each protein were averaged and standard deviations calculated for data presented in Figure 1, the average of the protein band intensities was normalized against a reference Ponceau Red band. 


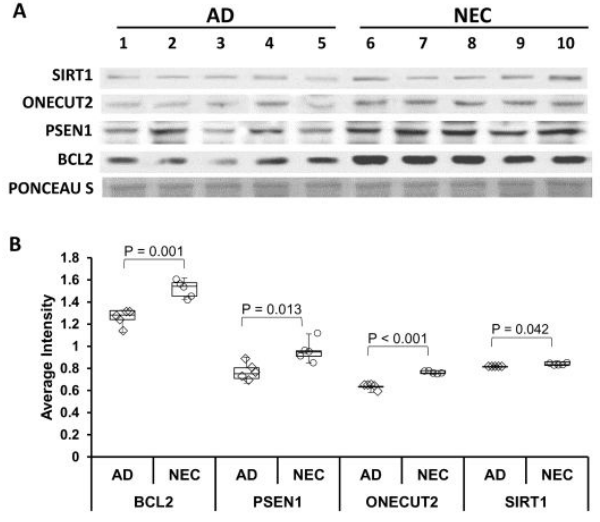

Figure 1: Levels of miR-34c-targeted proteins in $\mathrm{AD}$ and NEC plasma. Proteins were extracted from AD and NEC plasma and examined by Western blot. (A) Membranes were stained with Ponceau Red and probed for SIRT1, ONECUT2, PSEN1, and BCL2. (B) Relative intensities for each protein were calculated and presented in box plots. The bars indicate where multiple blots were spliced. Numbers 1-10 are used as sample identifiers.

We have previously shown that miR-34c is present in higher abundance in AD than in NEC plasma [6]. In this study, we examined linear trends to explore the correlation between expression of this miRNA and levels of its targets in $\mathrm{AD}$ and NEC plasma protein fraction (Figure 2). Statistically significant correlations between miR-34c levels and each target's abundance were found, with $p<0.001$ for ONECUT2, SIRT1 and PSEN1, and p=0.002 for BCL2 (Figure 2). This shows that increased miR-34c levels in AD are correlated with significantly decreased levels of all four targets in plasma.
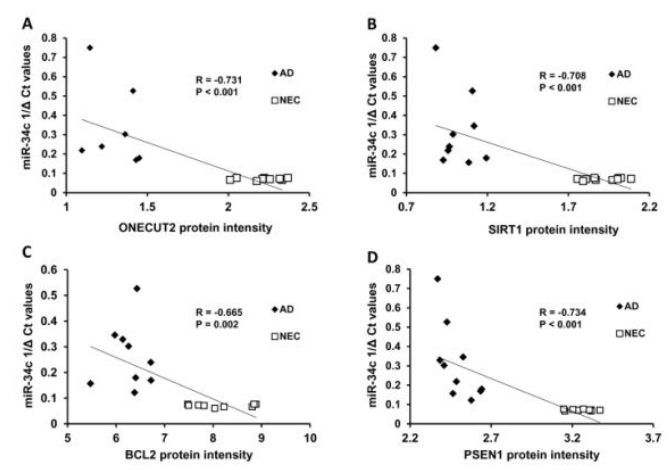

Figure 2: Relationships between miR-34c levels and protein expression in AD and NEC plasma. miR-34c levels were measured in $\mathrm{AD}$ and NEC plasma, and expressed as $1 / \Delta \mathrm{Ct}$. (A) These values were plotted against protein intensity for ONECUT2, (B) SIRT1, (C) BCL2 and (D) PSEN1. Pearson Correlation Value (R) and p values were calculated for each protein.

\section{Expression of ONECUT2, SIRT1, BCL2, and PSEN1 in MCI plasma compared to NEC and AD plasma}

Because we found a significantly decreased abundance of ONECUT2, SIRT1, BCL2, and PSEN1 in AD plasma compared to NEC plasma, we sought to determine whether these decreases in protein abundance could be detected earlier in disease progression, namely in the pre-dementia MCI phase. Therefore, we examined levels of these proteins in plasma from MCI participants, and compared expression to $\mathrm{AD}$ and NEC levels (Figure 3).

After normalization with Ponceau Red band intensities, all four proteins, BCL2, SIRT1, ONECUT2 and PSEN1, exhibited decreased expression when comparing AD or MCI individuals' plasma with NEC samples (Figures 3A-3D). Interestingly, while measurement of ONECUT2 circulating levels revealed a significant decrease in expression between NEC and MCI samples $(p<0.0001)$, there was no difference when comparing MCI and $\mathrm{AD}$ intensity levels (Figure $3 \mathrm{E}$ and Supplementary Table 1). This implies that decreased ONECUT2 may be manifested early in the disease process, and then may remain low as disease progresses.

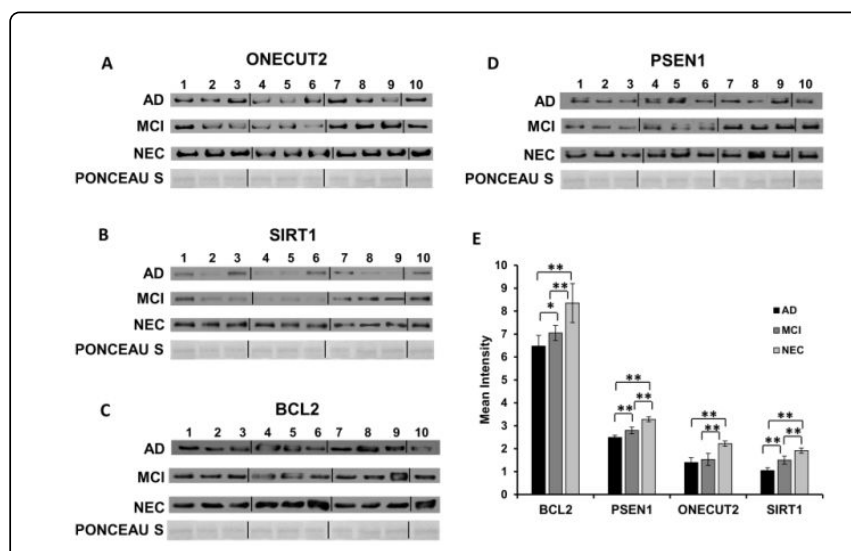

Figure 3: Expression of miRNA target proteins in $\mathrm{AD}, \mathrm{MCI}$ and NEC. Proteins were extracted from AD, MCI, and NEC plasma and examined by Western blot. (A) Membranes were stained with Ponceau Red, and probed for ONECUT2, (B) SIRT1, (C) BCL2 and (D) PSEN1. Intensities for each protein were calculated, averaged, and compared among AD, MCI, and (E) NEC samples. ${ }^{* *} \mathrm{p}<0.001$ and ${ }^{*} \mathrm{p}<0.05$. The bars indicate where multiple blots were spliced. Numbers 1-10 are used as sample identifiers.

Examination of SIRT1, BCL2, and PSEN1 plasma levels revealed that these proteins exhibit progressive decreases in expression from NEC to MCI, and again to the AD dementia stage of the illness (Figures 3A-3E and Supplementary Table 1). For both SIRT1 and PSEN1, $\mathrm{p}<0.0001$ when comparing NEC and MCI, NEC and AD, or $\mathrm{MCI}$ and $\mathrm{AD}$. For BCL2, $\mathrm{p}<0.0001$ comparing NEC and MCI; $<<0.001$ comparing NEC and $\mathrm{AD}$; and $\mathrm{p}=0.0169$ comparing $\mathrm{AD}$ and $\mathrm{MCI}$. These data indicate a pattern of stepwise repression of expression, in which these three proteins decrease in plasma during the transition from normalcy to mild cognitive impairment, and then again to the bona fide $\mathrm{AD}$ phase of cognitive decline. 

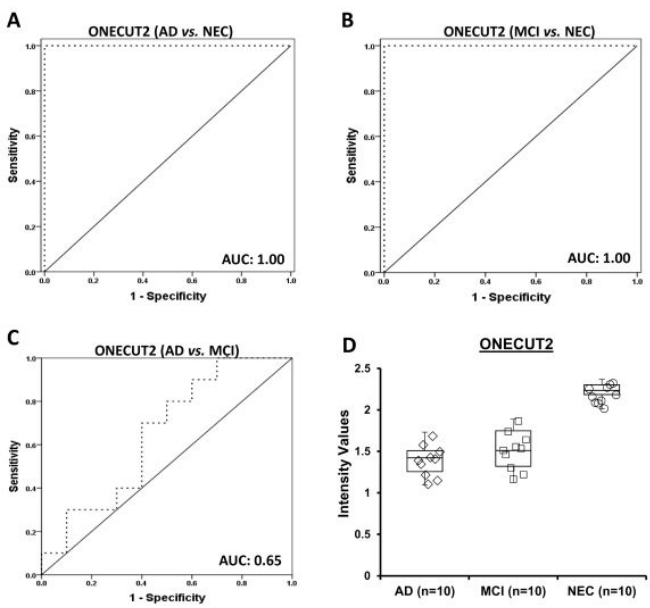

Figure 4: ONECUT2 distinguishes between NEC and AD/MCI. ROC analysis was performed for ONECUT2's ability to separate (A) AD vs. NEC, (B) MCI vs. NEC, and (C) AD vs. MCI. (D) Relative intensities of Onecut2 from individual plasma samples are displayed in a box plot.

\section{Plasma ONECUT2, BCL2, SIRT1, and PSEN1 as biomarkers of $\mathrm{AD}$ and MCI}

Because mean ONECUT2, BCL2, SIRT1, and PSEN1 levels are significantly lower in plasma from both $\mathrm{MCI}$ and $\mathrm{AD}$ patients (Figure 3 ) and inversely correlated with miR-34c levels (Figure 2), we next examined the ability of these proteins' levels to distinguish between the $\mathrm{AD}$ and NEC, MCI and NEC, and AD and MCI cohorts. Receiver operating characteristic (ROC) analysis was used to determine the sensitivity of the four target genes as biomarkers for the two different cognitive decline states. Our results show that ONECUT2 separates the $\mathrm{AD}$ and NEC groups as well as the MCI and NEC groups, with an area under the curve (AUC) of 1.0, indicating that ONECUT2 may serve as an excellent biomarker for differentiating both $\mathrm{AD}$ and $\mathrm{MCI}$ from NEC (Figures $4 \mathrm{~A}$ and $4 \mathrm{~B}$ and Supplementary Table 2). However, because ONECUT2 levels remain low throughout progression from $\mathrm{MCI}$ to $\mathrm{AD}$, this protein performs poorly as a biomarker to distinguish between these two disease states with an AUC value of only 0.65 (Figure 4C and Supplementary Table 2).

The same statistical analysis revealed a different pattern of biomarker efficacy for the other three proteins. Plasma levels of SIRT1, BCL2, and PSEN1 differentiate between NEC and AD, NEC and MCI, and $\mathrm{AD}$ and $\mathrm{MCI}$, with excellent AUC values (Figures 5A-5C and Supplementary Table 2). When distinguishing between NEC and AD, all three target proteins demonstrated an AUC of 1.0. For differentiating between the MCI and NEC groups, BCL2 had an AUC of 0.96, SIRT1 an AUC of 0.99, and PSEN1 an AUC of 1.0. In separating the $\mathrm{AD}$ and MCI groups, the AUC for BCL2 was 0.91 and 1.0 for both SIRT1 and PSEN1. Together, these data indicate that all three of these proteins may serve as robust biomarkers for differentiating between the three stages of disease progression.

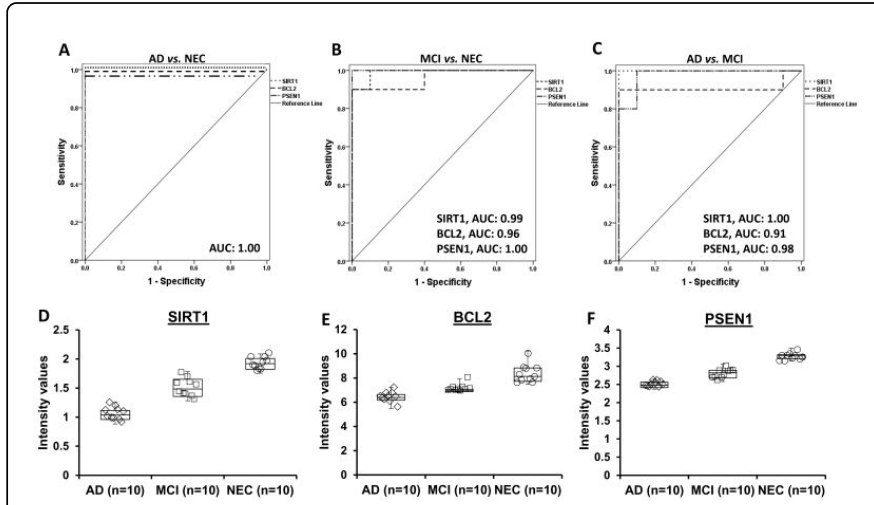

Figure 5: BCL2, SIRT1, and PSEN1 distinguish among NEC, MCI and $\mathrm{AD}$ groups. ROC analysis was performed to determine the ability of BCL2, SIRT1, and PSEN1 to distinguish between (A) AD vs. NEC, (B) MCI vs. NEC, and (C) AD vs. MCI. Comparison between the two different states by BCL2 is represented by dotted lines, by SIRT1 is represented by ----, and by PSEN1 by lines. (D) Relative intensities of SIRT1, (E) BCL2, and (F) PSEN1 from individual plasma samples are displayed in box plots.

\section{Inverse relationship between miR-34c and miR-34a and protein expression in $\mathrm{AD}, \mathrm{MCI}$, and NEC plasma}
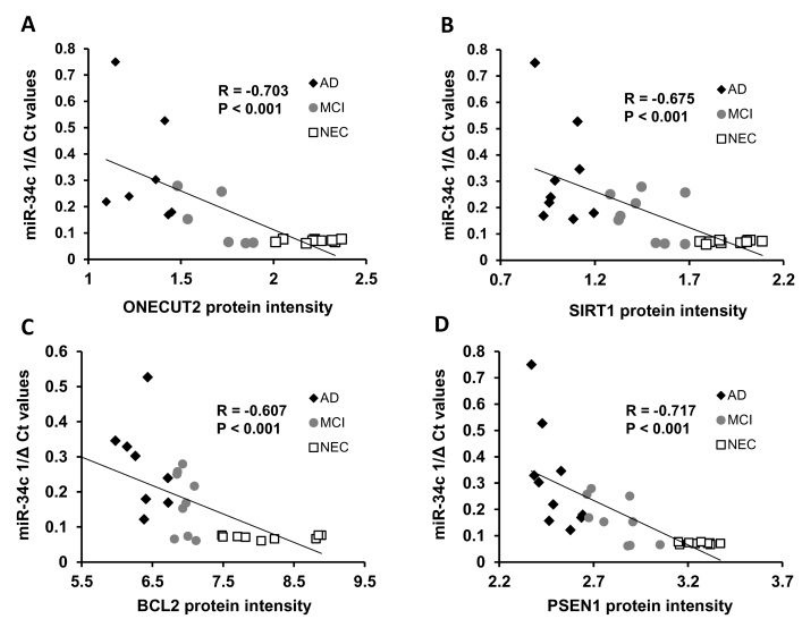

Figure 6: Relationships between miR-34c levels and protein expression. miR-34c levels were measured in $\mathrm{AD}, \mathrm{MCI}$, and NEC plasma, and expressed as $1 / \Delta C$ t. These values were plotted against protein intensity for (A) ONECUT2, (B) SIRT1, (C) BCL2, and (D) PSEN1. Pearson Correlation Value $(\mathrm{R})$ and $\mathrm{p}$ values were calculated for each protein.

Finally, we examined linear trends to explore the correlation between expression of miR-34c and miR-34a and down-regulation of their protein targets in AD, MCI, and NEC plasma (Figures 6 and 7). Statistically significant correlations between miR-34c levels and each target protein were found, with $\mathrm{p}<0.001$ for all four proteins examined (Figure 6). Analysis of the relationships between miR-34a and target proteins shows the inverse relationship to be significant only for 
Onecut2 $(\mathrm{p}=0.005)$ (Figure 7A) but not the other three targets (Figures 7B-7D). This shows that among all four targets, decreased ONECUT2 level is most significantly correlated with the increase of both miR-34c and miR34a levels comparing the plasma specimens of all three groups.
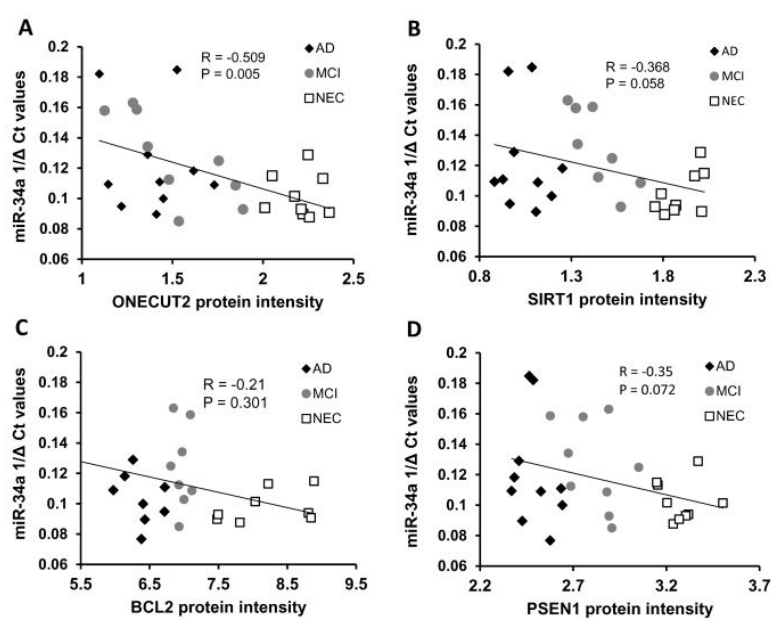

Figure 7: Relationships between miR-34a levels and protein expression. miR-34a levels were measured in $\mathrm{AD}, \mathrm{MCI}$, and NEC plasma, and expressed as $1 / \Delta \mathrm{Ct}$. These values were plotted against (A) protein intensity for ONECUT2, (B) SIRT1, (C) BCL2, and (D) PSEN1. Pearson Correlation Value (R) and $p$ values were calculated for each protein.

\section{Discussion}

In this study, we show that known target genes, ONECUT2, BCL2, SIRT1, and PSEN1, of two microRNAs, miR-34a \& -34c, are significantly reduced in abundance in plasma from $\mathrm{AD}$ and $\mathrm{MCI}$ patients as compared to NEC samples. Additionally, these reduced protein abundance levels correspond to the increased presence of the circulating miR-34c levels in examined plasma samples. Specifically, we demonstrate here that decreased ONECUT2 expression in plasma is a powerful and novel biomarker to distinguish normal elderly controls from MCI and AD patients, while BCL2, SIRT1, and PSEN1 distinguish among all three stages, from normalcy to the pre-dementia (MCI) and dementia (AD) stages of cognitive decline.

Selection of the four proteins included in our study follows the findings that they are targets of two microRNAs, miR-34a and -34c, as previously reported by cell-based functional assays [6]. Determination of a specific protein to be a good plasma biomarker is largely based on receiver operating characteristic (ROC) analysis. The specific area under the curve (AUC) calculated by the ROC method indicates whether a specific protein target of these two microRNAs is a good or poor protein biomarker. For example, Onecut2 is a powerful biomarker to distinguish the MCI state from normalcy, since the AUC value is 1.0 (Figure $4 \mathrm{~B}$ ). However, this protein's abundance is not a good biomarker to distinguish $\mathrm{MCI}$ from the $\mathrm{AD}$ state, due to a low AUC value of 0.65 (Figure 4C). Figure 8 provides a flow chart outlining the identification and classification process from the qPCR analysis of the two microRNAs' levels, to Western blotting of the abundance of their targets in the same individuals, to ROC analysis for the AUC values.

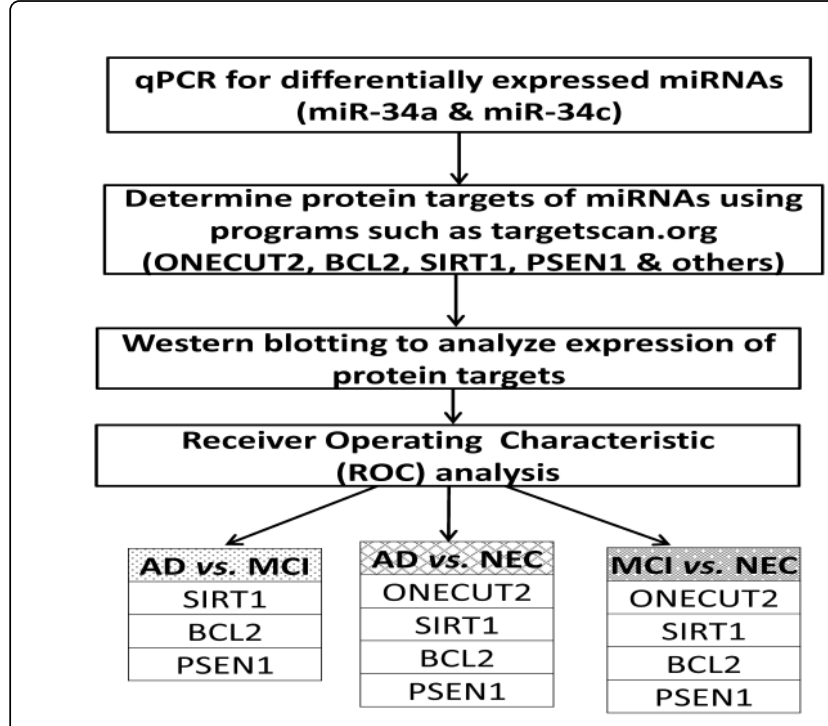

Figure 8: A flow chart summarizing the process of identifying and classifying plasma protein biomarkers for $\mathrm{AD}$ and MCI. Based on qPCR data for miR-34a and miR-34c, gene targets are identified and measured through Western blotting. ROC analysis is then applied to assess the ability of each protein to distinguish between different disease states.

These decreases may have important physiological implications in MCI and AD (Figure 9). The inverse relationship between miR-34c abundance and levels of its protein targets may be thought of as a balance between two families of molecules, with various degrees of tilting between miR-34c and ONECUT2 in normal elderly controls, $\mathrm{MCI}$, and $\mathrm{AD}$ stages (Figures 9A1-9A3). In this miRNA-target relationship, the degree of repression of the ONECUT2 gene reaches its maximal level at the transition between normal aging and MCI. In contrast, the other three targets of miR-34c, i.e. SIRT1, BCL2, and PSEN1, tilt more gradually from NEC to MCI, and then to AD, with the most severe tilting and lowest protein levels seen in the AD phase (Figures 9B1-9B3). Thus, the inverse relationship between miR-34c levels and expression of its targets, as represented here by four proteins, may point to a miR/target partnership in the control of $\mathrm{AD}$ pathogenesis, including the processes of amyloid plaque formation, synaptic decline, and neuronal apoptosis (Figures 9C1-9C3).

Our observation that increased circulating miRNA expression correlates with decreased circulating target protein expression suggests that plasma may provide a window into the etiopathogenesis of CNS disorders. Plasma, which is more readily available and easily accessible than other fluids such as cerebrospinal fluid, can therefore be reliably used to investigate interactions between miRNA and protein expression in neurodegeneration, and perhaps other diseases as well. Indeed, SIRT1 expression is down-regulated in both cortex of $\mathrm{AD}$ patients [60] and serum from AD and MCI patients [61], suggesting that circulating SIRT1 concentrations reflect levels in diseased tissue. Along with the current study, these results indicate that circulating protein and miRNA changes reflect pathological changes in tissue, and that plasma is a viable source of clinically-accessible protein biomarkers. 
Intriguingly, our data point to ONECUT2 as a potentially important and novel circulating protein in neurodegenerative progression. Although several studies demonstrate a role for ONECUT2 as a transcription factor involved in liver [62] and neurological development $[9,10]$, no link between this transcription factor and AD has previously been established. However, some brain and spinal structures influenced by ONECUT2 expression may also be involved in $\mathrm{AD}$ pathology. Of particular interest, the locus coeruleus is implicated as a prime contributor to inflammation, metabolic changes, and breakdown of the blood-brain barrier early in $\mathrm{AD}$ development [63-65]. Here we observe that ONECUT2, a driver of locus coeruleus development [7], significantly decreases fairly early in disease progression, i.e. at the MCI stage. This suggests that circulating ONECUT2 expression, regulated by both miR-34c and miR-34a, may serve as an early warning signal of AD-related processes in this key brain region.

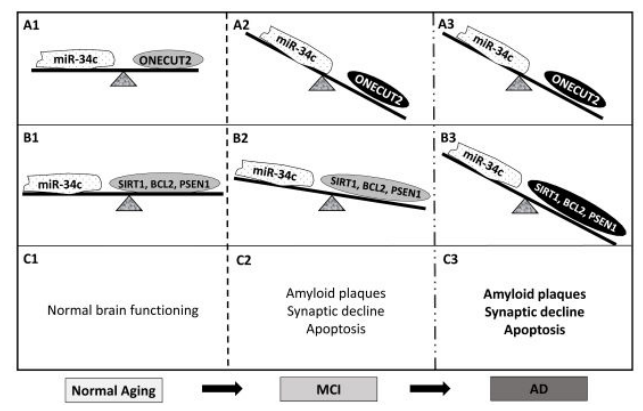

Figure 9: Relationships between miR-34c, target protein expression, and pathological processes during disease progression. In normal elderly controls, miR-34c and target protein levels are normal and balanced, and the brain functions normally (A1, B1, C1). As disease progresses to the MCI state, miR-34c expression increases, resulting in Onecut2 expression decreasing rapidly and SIRT1, BCL2, and PSEN1 abundance decreasing at a lower rate, leading to early stages of amyloid plaque deposition, synaptic decline, and neuronal apoptosis (A2, B2, and $\mathrm{C} 2$ ). Once the $\mathrm{AD}$ stage is reached, ONECUT2 is at similarly low levels as in the MCI phase, but miR-34c levels are even higher than in the MCI phase; SIRT1, BCL2, and PSEN1 expression has decreased from the MCI stage; amyloid plaque deposition, synaptic decline, and neuronal apoptosis continue (A3, B3, and C3).

Additionally, because ONECUT2 levels are expressed at significantly reduced levels early in disease progression, this protein may best serve as a prognostic indicator of very early cognitive decline. Indeed, it may point to the possibility of early therapeutic intervention and delay of disease progression. On the other hand, because BCL2, SIRT1, and PSEN1 expression all decrease both during the transition from NEC to MCI and even further during the transition from MCI to $\mathrm{AD}$, these proteins may be useful as non-invasive diagnostic tools for differentiating between various stages of cognitive decline. Patients with MCI-range plasma levels of these three proteins may be targeted for therapy in an effort to arrest or delay transition to full-blown AD.

Although we have already identified miR-34c as a principal biomarker of $\mathrm{AD}$ and regulator of ONECUT2, BCL2, SIRT1, and PSEN1, a host of other miRNAs target these proteins as well. Taken together, these miRNAs and our lead miRNAs, miR-34c and miR-34a, may comprise a multifaceted disease signature, in which up-regulation of various combinations of miRNAs may indicate differential risk for disease onset and conversion to $\mathrm{AD}$. Further experiments must be completed to examine these miRNAs in $\mathrm{AD}$ and MCI plasma. Additionally, although the current study used a small sample size, our results point to a general trend of decreased target protein expression in $\mathrm{AD}$ and MCI. It will now be essential to conduct larger scale enzyme-linked immunosorbent assay (ELISA) experiments, in which protein levels are examined in plasma from significantly bigger cohort studies with $\mathrm{AD}$ and $\mathrm{MCI}$ patients, as well as in individuals with non$\mathrm{AD}$ dementias.

In conclusion, we demonstrate decreased plasma expression of miR-34c target proteins, with ONECUT2 emerging as a potential candidate biomarker for transition from normal states to mild cognitive impairment (MCI), while the levels of SIRT1, BCL2, and PSEN1 may serve as diagnostic markers for further transition of decline from MCI to bonafide AD. Further work needs to be done to examine levels of other miR-34c-targeted proteins and to validate these proteins as prognostic and diagnostic biomarkers in large-cohort studies.

\section{Acknowledgements}

The authors would like to thank Mr. Alan N. Bloch for proofreading the manuscript; Dr. Zongfei Yuan for his work in performing the miR-34c qPCR assays; Samantha Jenkins for performing the Western blotting; and Shelley Solomon, Victor Whitehead, Chris Hosein, Orchid Lin, Carmela Galindez and the Memory Clinic staff for coordinating the clinical blood collections. This work was supported by grants [AG044157 and AG035410] from the National Institute on Aging of the National Institutes of Health to EW.

\section{Disclosure Statement}

Eugenia Wang is on entrepreneurial leave from the University of Louisville School of Medicine, with 51\% of her effort committed to Advanced Genomic Technology, LLC, a start-up company in Louisville, Kentucky; her other $49 \%$ is at the University of Louisville, as the Gheens Endowed Chair on Aging and Professor of Biochemistry and Molecular Biology. Arin Zirnheld and Vikranth Shetty are employees of Advanced Genomic Technology (AGT), and Erik L Regalado was also an employee of AGT at the time the experiments were performed.

Howard Chertkow sits on an adjudication board for clinical trials for Bristol Myers Squibb, and has been a speaker and Advisory Board member for Pfizer Canada. Hyman Schipper has served as consultant to Caprion Pharmaceuticals, Molecular Biometrics Inc., Osta Biotechnologies, TEVA Neurosciences, Immunotec, and Apopharma.

\section{References}

1. Ferri CP, Prince M, Brayne C, Brodaty H, Fratiglioni L, et al. (2005) Global prevalence of dementia: a Delphi consensus study. Lancet 366: 2112-2117.

2. Bossy-Wetzel E, Schwarzenbacher R, and Lipton SA (2004) Molecular pathways to neurodegeneration. Nat Med 10: S2-S9.

3. Nizzari M, Thellung S, Corsaro A, Villa V, Pagano A, et al. (2012) Neurodegeneration in Alzheimer disease: role of amyloid precursor protein and presenilin 1 intracellular signaling. J Toxicol 2012: 187297.

4. Shimohama S (2000) Apoptosis in Alzheimer's disease -- an update. Apoptosis Int J Program Cell Death 5: 9-16. 
5. Li X, Khanna A, Li N, Wang E (2011) Circulatory miR34a as an RNAbased, noninvasive biomarker for brain aging. Aging (Albany NY) 3: 985-1002.

6. Bhatnagar S, Chertkow H, Schipper HM, Yuan Z, Shetty V, et al. (2014) Increased microRNA-34c abundance in Alzheimer's disease circulating blood plasma. Front Mol Neurosci 7: 2.

7. Espana A, Clotman F (2012) Onecut factors control development of the Locus Coeruleus and of the mesencephalic trigeminal nucleus. Mol Cell Neurosci 50: 93-102.

8. Espana A, Clotman F (2012) Onecut transcription factors are required for the second phase of development of the A13 dopaminergic nucleus in the mouse. J Comp Neurol 520: 1424-1441.

9. Francius C, Clotman F (2010) Dynamic expression of the Onecut transcription factors HNF-6, OC-2 and OC-3 during spinal motor neuron development. Neuroscience 165: 116-129.

10. Hodge LK, Klassen MP, Han BX, Yiu G, Hurrell J, et al. (2007) Retrograde BMP signaling regulates trigeminal sensory neuron identities and the formation of precise face maps. Neuron 55: 572-586.

11. Stam FJ, Hendricks TJ, Zhang J, Geiman EJ, Francius C, et al. (2012) Renshaw cell interneuron specialization is controlled by a temporally restricted transcription factor program. Development 139: 179-190.

12. Araki T, Sasaki Y, Milbrandt J (2004) Increased nuclear NAD biosynthesis and SIRT1 activation prevent axonal degeneration. Science 305: 1010-1013.

13. Di Emidio G, Falone S, Vitti M, D'Alessandro AM, Vento M, et al. (2014) SIRT1 signalling protects mouse oocytes against oxidative stress and is deregulated during aging. Hum Reprod 29: 2006-2017.

14. Gueguen C, Palmier B, Plotkine M, Marchand-Leroux C, and Besson VC (2014) Neurological and histological consequences induced by in vivo cerebral oxidative stress: Evidence for beneficial effects of SRT1720, a Sirtuin 1 activator, and Sirtuin 1-mediated neuroprotective effects of poly(ADP-ribose) polymerase inhibition. PLoS ONE 9: e87367.

15. Huang B, Cheng X, Wang D, Peng M, Xue Z, et al. (2014) Adiponectin promotes pancreatic cancer progression by inhibiting apoptosis via the activation of AMPK/Sirt1/PGC-1 $\alpha$ signaling. Oncotarget 5: 4732-4745.

16. Khan RS, Dine K, Das Sarma J, and Shindler KS (2014) SIRT1 activating compounds reduce oxidative stress mediated neuronal loss in viral induced CNS demyelinating disease. Acta Neuropathol Commun 2: 3 .

17. Lee HR, Shin HK, Park SY, Kim HY, Lee WS, et al. (2014) Attenuation of $\beta$-amyloid-induced tauopathy via activation of CK2 $\alpha \pm / S I R T 1$ : targeting for cilostazol. J Neurosci Res 92: 206-217.

18. Sirotkin AV, Dekanova P, Harrath AH, Alwasel SH, Vašiček D (2014) Interrelationships between sirtuin 1 and transcription factors p53 and NFKB (p50/p65) in the control of ovarian cell apoptosis and proliferation. Cell Tissue Res 358: 627-632.

19. Sun $\mathrm{Q}, \mathrm{Hu} H$, Wang $W$, Jin $H$, Feng G, et al. (2014) Taurine attenuates amyloid $\beta$ 1-42-induced mitochondrial dysfunction by activating of SIRT1 in SK-N-SH cells. Biochem Biophys Res Commun 447: 485-489.

20. Yao H, Sundar IK, Ahmad T, Lerner C, Gerloff J, et al. (2014) SIRT1 protects against cigarette smoke-induced lung oxidative stress via a FOXO3-dependent mechanism. Am J Physiol Lung Cell Mol Physiol 306 L816-828.

21. Nunez G, Seto M, Seremetis S, Ferrero D, Grignani F, et al. (1989) Growth- and tumor-promoting effects of deregulated BCL2 in human Blymphoblastoid cells. Proc Natl Acad Sci U S A 86: 4589-4593.

22. Abe-Dohmae S, Harada N, Yamada K, Tanaka R (1993) Bcl-2 gene is highly expressed during neurogenesis in the central nervous system. Biochem Biophys Res Commun 191: 915-921.

23. Merry DE, Veis DJ, Hickey WF, Korsmeyer SJ (1994) bcl-2 protein expression is widespread in the developing nervous system and retained in the adult PNS. Development 120:301-311.

24. Imaizumi K, Morihara T, Mori Y, Katayama T, Tsuda M, et al. (1999) The cell death-promoting gene DP5, which interacts with the BCL2 family, is induced during neuronal apoptosis following exposure to amyloid beta protein. J Biol Chem 274: 7975-7981.
25. Paradis E, Douillard H, Koutroumanis M, Goodyer C, LeBlanc A (1996) Amyloid beta peptide of Alzheimer's disease downregulates Bcl-2 and upregulates bax expression in human neurons. J Neurosci 16: 7533-7539.

26. Ferreiro E, Eufrásio A, Pereira C, Oliveira CR, Rego AC (2007) Bcl-2 overexpression protects against amyloid-beta and prion toxicity in GT1-7 neural cells. J Alzheimers Dis 12: 223-228.

27. De Strooper B, Saftig P, Craessaerts K, Vanderstichele H, Guhde G, et al. (1998) Deficiency of presenilin-1 inhibits the normal cleavage of amyloid precursor protein. Nature 391: 387-390.

28. Sorbi S, Nacmias B, Forleo P, Piacentini S, Sherrington R, et al. (1995) Missense mutation of S182 gene in Italian families with early-onset Alzheimer's disease. Lancet 346: 439-440.

29. Tanahashi $\mathrm{H}$, Mitsunaga $\mathrm{Y}$, Takahashi $\mathrm{K}$, Tasaki $\mathrm{H}$, Watanabe $\mathrm{S}$, et al. (1995) Missense mutation of S182 gene in Japanese familial Alzheimer's disease. Lancet 346: 440.

30. Sassi C, Guerreiro R, Gibbs R, Ding J, Lupton MK, et al. (2014) Investigating the role of rare coding variability in Mendelian dementia genes (APP, PSEN1, PSEN2, GRN, MAPT, and PRNP) in late-onset Alzheimer's disease. Neurobiol Aging 35: 2881.

31. Yesavage JA, Brink TL, Rose TL, Lum O, Huang V, et al. (1982) Development and validation of a geriatric depression screening scale: a preliminary report. J Psychiatr Res 17: 37-49.

32. Hachinski VC, Iliff LD, Zilhka E, Du Boulay GH, McAllister VL, et al. (1975) Cerebral blood flow in dementia. Arch Neurol 32: 632-637.

33. Nasreddine ZS, Phillips NA, Bédirian V, Charbonneau S, Whitehead V, et al. (2005) The Montreal Cognitive Assessment, MoCA: a brief screening tool for mild cognitive impairment. J Am Geriatr Soc 53: 695-699.

34. Folstein MF, Folstein SE, McHugh PR (1975) "Mini-mental state". A practical method for grading the cognitive state of patients for the clinician. J Psychiatr Res 12: 189-198.

35. McKhann G, Drachman D, Folstein M, Katzman R, Price D, et al. (1984) Clinical diagnosis of Alzheimer's disease: report of the NINCDS-ADRDA Work Group under the auspices of Department of Health and Human Services Task Force on Alzheimer's Disease. Neurology 34: 939-944.

36. Reisberg B, Ferris SH, de Leon MJ, Crook T (1982) The Global Deterioration Scale for assessment of primary degenerative dementia. Am J Psychiatry 139: 1136-1139.

37. Lawton MP, Brody EM (1969) Assessment of older people: selfmaintaining and instrumental activities of daily living. Gerontologist 9: 179-186.

38. Winblad B, Palmer K, Kivipelto M, Jelic V, Fratiglioni L, et al. (2004) Mild cognitive impairment--beyond controversies, towards a consensus: report of the International Working Group on Mild Cognitive Impairment. J Intern Med 256: 240-246.

39. Petersen RC, Smith GE, Ivnik RJ, Tangalos EG, Schaid DJ, et al. (1995) Apolipoprotein E status as a predictor of the development of Alzheimer's disease in memory-impaired individuals. JAMA 273: 1274-1278.

40. Petersen RC, Smith GE, Waring SC, Ivnik RJ, Tangalos EG, et al. (1999) Mild cognitive impairment: clinical characterization and outcome. Arch Neurol 56: 303-308.

41. Darvesh S, Leach L, Black SE, Kaplan E, Freedman M (2005) The behavioural neurology assessment. Can J Neurol Sci 32: 167-177.

42. Petersen RC (2004) Mild cognitive impairment as a diagnostic entity. J Intern Med 256: 183-194.

43. American Psychiatric Association (1994) Diagnostic and Statistical Manual of Mental Disorders (4thedn.) DSM-IV. Washington, D.C.

44. Wechsler D (1981) Wechsler Adult Intelligence Scale - Revised. The Psychological Corporation, New York

45. Wechsler D (1987) Wechsler Memory Scale - Revised. The Psychological Corporation, San Antonio

46. Raven JC (1947) Colored Progressive Matrices Sets A, Ab, B. H.K. Lewis, London 
Citation: Zirnheld AL, Regalado EL, Shetty V, Chertkow H, Schipper HM, et al. (2015) Target Genes of Circulating miR-34c as Plasma Protein Biomarkers of Alzheimer's Disease and Mild Cognitive Impairment. Aging Sci 3: 140. doi:10.4172/2329-8847.1000140

Page 9 of 9

47. Benton AL, Sivan AB, Hamsher K, Varney N, Spreen O (1983) Contributions to Neuropsychological Assessment. A Clinical Manual. Oxford University Press, New York

48. Bondareff W, Mountjoy CQ, Roth M, Rossor MN, Iversen LL, et al. (1987) Age and histopathologic heterogeneity in Alzheimer's disease. Evidence for subtypes. Arch Gen Psychiatry 44: 412-417.

49. Rey A (1941) L'examen psychologique dans les cas d'encéphalopathie traumatique. (Les problems.). [The psychological examination in cases of traumatic encephalopathy. Problems.]. Arch Psychol 28: 215-285

50. Chomczynski P (1993) A reagent for the single-step simultaneous isolation of RNA, DNA and proteins from cell and tissue samples. Biotechniques 15: 532-534, 536-7.

51. Aldridge GM, Podrebarac DM, Greenough WT, Weiler IJ (2008) The use of total protein stains as loading controls: an alternative to high abundance single-protein controls in semi-quantitative immunoblotting. J Neurosci Methods 172: 250-254.

52. Gilda JE, Gomes AV (2013) Stain-Free total protein staining is a superior loading control to $\beta$ actin for Western blots. Anal Biochem 440: 186-188.

53. Dittmer A, Dittmer J (2006) Beta-actin is not a reliable loading control in Western blot analysis. Electrophoresis 27: 2844-2845.

54. Romero-Calvo I, Ocón B, Martínez-Moya P, Suárez MD, Zarzuelo A, et al. (2010) Reversible Ponceau staining as a loading control alternative to actin in Western blots. Anal Biochem 401: 318-320.

55. Hayter AJ (1986) The Maximum Familywise Error Rate of Fisher's Least Significant Difference Test. J Am Stat Assoc 81: 1000.

56. Zweig MH, Campbell G (1993) Receiver-operating characteristic (ROC) plots: a fundamental evaluation tool in clinical medicine. Clin Chem 39: 561-577.

57. Hajian-Tilaki K (2013) Receiver Operating Characteristic (ROC) Curve Analysis for Medical Diagnostic Test Evaluation. Caspian J Intern Med 4: 627-635.
58. Florkowski CM (2008) Sensitivity, specificity, receiver-operating characteristic (ROC) curves and likelihood ratios: communicating the performance of diagnostic tests. Clin Biochem Rev 29 Suppl 1: S83-87.

59. Strittmatter WJ, Saunders AM, Schmechel D, Pericak-Vance M, Enghild J, et al. (1993) Apolipoprotein E: high-avidity binding to beta-amyloid and increased frequency of type 4 allele in late-onset familial Alzheimer disease. Proc Natl Acad Sci U S A 90: 1977-1981.

60. Julien C, Tremblay C, Emond V, Lebbadi M, Salem N Jr, et al. (2009) Sirtuin 1 reduction parallels the accumulation of tau in Alzheimer disease. J Neuropathol Exp Neurol 68: 48-58.

61. Kumar R, Chaterjee P, Sharma PK, Singh AK, Gupta A, et al. (2013) Sirtuin1: a promising serum protein marker for early detection of Alzheimer's disease. PLoS One 8: e61560.

62. Clotman F, Jacquemin P, Plumb-Rudewiez N, Pierreux CE, Van der Smissen P, et al. (2005) Control of liver cell fate decision by a gradient of TGF beta signaling modulated by Onecut transcription factors. Genes Dev 19: 1849-1854.

63. Jacobs HI, Wiese S, van de Ven V, Gronenschild EH, Verhey FR, et al. (2015) Relevance of parahippocampal-locus coeruleus connectivity to memory in early dementia. Neurobiol Aging 36: 618-626.

64. Liu L, Luo S, Zeng L, Wang W, Yuan L, et al. (2013) Degenerative alterations in noradrenergic neurons of the locus coeruleus in Alzheimer's disease. Neural Regen Res 8: 2249-2255.

65. Mravec B, Lejavova K, Cubinkova V (2014) Locus (coeruleus) minoris resistentiae in pathogenesis of Alzheimer's disease. Curr Alzheimer Res 11: $992-1001$. 